\title{
IMF-METALLICITY: A TIGHT LOCAL RELATION REVEALED BY THE CALIFA SURVEY
}

\author{
Ignacio Martín-Navarro ${ }^{1,2}$, Alexandre VAzdekis ${ }^{1,2}$, Francesco La Barbera $^{3}$, Jesús Falcón-Barroso ${ }^{1,2}$, \\ Mariya Lyubenova $^{4}$, Glenn van de Ven ${ }^{5}$, Ignacio Ferreras ${ }^{6}$, S. F. SÁnchez ${ }^{7}$, S. C. Trager ${ }^{4}$, R. García-Benito ${ }^{8}$, D. Mast ${ }^{9}$, \\ M. A. Mendoza ${ }^{8}$, P. Sánchez-Blázquez ${ }^{10}$, R. González Delgado ${ }^{8}$, and C. J. Walcher ${ }^{11}$ \\ (The CALIFA TeAm) \\ ${ }^{1}$ Instituto de Astrofísica de Canarias, c/Vía Láctea s/n, E-38205—La Laguna, Tenerife, Spain; imartin@iac.es \\ ${ }^{2}$ Departamento de Astrofísica, Universidad de La Laguna, E-38205 La Laguna, Tenerife, Spain \\ ${ }^{3}$ INAF-Osservatorio Astronomico di Capodimonte, Napoli, Italy \\ ${ }^{4}$ Kapteyn Astronomical Institute, University of Groningen, Postbus 800, 9700 AV Groningen, The Netherlands \\ ${ }_{5}$ Max Planck Institute for Astronomy, Königstuhl 17, D-69117 Heidelberg, Germany \\ ${ }^{6}$ Mullard Space Science Laboratory, University College London, Holmbury St Mary, Dorking, Surrey RH5 6NT, UK \\ ${ }^{7}$ Instituto de Astronomía, Universidad Nacional Autonóma de México, A.P. 70-264, 04510 México, D.F., México \\ ${ }^{8}$ Instituto de Astrofísica de Andalucía (CSIC), Glorieta de la Astronomía s/n, Aptdo. 3004, E-18080 Granada, Spain \\ ${ }^{9}$ Instituto de Cosmologia, Relatividade e Astrofísica-ICRA, Centro Brasileiro de Pesquisas Físicas, \\ Rua Dr.Xavier Sigaud 150, CEP 22290-180, Rio de Janeiro, RJ, Brazil \\ ${ }^{10}$ Departamento de Física Teórica, Universidad Autónoma de Madrid, Cantoblanco, E-28049 Spain \\ ${ }^{11}$ Leibniz-Institut für Astrophysik Potsdam (AIP), An der Sternwarte 16, D-14482 Potsdam, Germany \\ Received 2015 April 24; accepted 2015 May 30; published 2015 June 18
}

\begin{abstract}
Variations in the stellar initial mass function (IMF) have been invoked to explain the spectroscopic and dynamical properties of early-type galaxies (ETGs). However, no observations have yet been able to disentangle the physical driver. We analyze here a sample of 24 ETGs drawn from the CALIFA survey, deriving in a homogeneous way their stellar population and kinematic properties. We find that the local IMF is tightly related to the local metallicity, becoming more bottom-heavy toward metal-rich populations. Our result, combined with the galaxy mass-metallicity relation, naturally explains previous claims of a galaxy mass-IMF relation, derived from non-IFU spectra. If we assume that—within the star formation environment of ETGs-metallicity is the main driver of IMF variations, a significant revision of the interpretation of galaxy evolution observables is necessary.
\end{abstract}

Key words: galaxies: elliptical and lenticular, cD - galaxies: evolution - galaxies: formation galaxies: fundamental parameters - galaxies: stellar content

\section{INTRODUCTION}

The stellar initial mass function (IMF) determines the ratio between dwarf and giant stars, and therefore, ultimately regulates the chemical enrichment and the stellar feedback. Moreover, it is the Rosetta stone in our understanding of unresolved stellar systems, relating the properties of resolved nearby stars to the integrated light of more distant objects.

Over the last years, there has been growing evidence of a variable IMF, as opposed to the common assumption that the IMF of the Milky Way is universal (Kroupa 2002; Bastian et al. 2010). These claims come from a wide variety of approaches, including stellar population analysis (Cenarro et al. 2003; van Dokkum \& Conroy 2010; Spiniello et al. 2012; Ferreras et al. 2013), gravitational lensing (Treu et al. 2010; Thomas et al. 2011), and dynamical models (Cappellari et al. 2012).

Assuming that the answer to all of these observational results is indeed a non-universal IMF, two main parameters have been proposed. On the one hand, the integrated stellar velocity dispersion is found to correlate with the IMF slope as inferred from both dynamical models (e.g., Cappellari et al. 2012) and stellar population analysis (e.g., La Barbera et al. 2013). On the other hand, Conroy \& van Dokkum (2012) claimed a stronger correlation when comparing the $[\mathrm{Mg} / \mathrm{Fe}]$ pattern to the IMF slope. Nevertheless, these trends have to be carefully interpreted because they are based on the spatially integrated light of early-type galaxies (ETGs). Thus, the inferred properties are luminosity weighted quantities, convolved by the radial light distribution, which depends itself on the stellar velocity dispersion (Graham et al. 2001), and is highly peaked toward the center.

We showed in Martín-Navarro et al. (2015a, 2015b, see also Pastorello et al. 2014) that the IMF is a local property, not only varying among but also within galaxies. The lack of a large statistical sample, however, limited further interpretations of our data, and the question about which is the main driver of IMF variations remains open. In this Letter, we use the 2D spatially resolved IFU data from the CALIFA survey (Sánchez et al. 2012) to address this problem by analyzing the radial IMF profiles of a sample of 24 ETGs.

\section{DATA}

We selected the sub-sample of 24 ETGs with redshift $0.018<z<0.030$ among the observed CALIFA galaxies (for sample properties, see Walcher et al. 2014). In the local universe, the strong IMF sensitive index $\mathrm{TiO}_{2}$ is potentially affected by a telluric absorption feature at $\sim 6280 \AA$. The selected redshift window maximizes the number of ETGs unaffected by this feature. Technical details of the data are fully described in Sánchez et al. (2012) and García-Benito et al. (2014). The central velocity dispersion in our galaxy sample ranges from $\sim 160$ to $\sim 310 \mathrm{~km} \mathrm{~s}^{-1}$, although the bulk of them (21 galaxies) have velocity dispersions greater than $200 \mathrm{~km} \mathrm{~s}^{-1}$. The mean stellar mass in our sample is $\bar{M}_{\star}=10^{11.54} M_{\odot}$ according to González Delgado et al. (2014). 
We derived the line of sight mean stellar velocity $(V)$ and velocity dispersion $(\sigma)$ following J. Falcón-Barroso et al. (2015, in preparation) Using these measurements, each IFU spaxel was then corrected to the rest-frame. Finally, we radially binned each galaxy using elliptical apertures. The size of the apertures was set to reach a signal-to-noise of, at least, $125 \AA^{-1}$ at $\lambda \lambda=6000,6200 \AA$. All the quantities discussed in this Letter are averaged spaxel-values over these elliptical annuli.

\section{ANALYSIS}

\subsection{Stellar Populations}

For this work, we made use of the extended MILES (MIUSCAT) stellar population models (Vazdekis et al. 2010, 2012). We assume a bimodal, low-mass tapered, IMF, whose only free parameter, $\Gamma_{b}$, is the slope of the high-mass end (above $0.6 M_{\odot}$ ) of the distribution. This parametrization, first introduced by Vazdekis et al. (1996), generalizes the Kroupa IMF, which is recovered for $\Gamma_{b}=1.35$. Note that the current version of the extended MILES models cover from $\Gamma_{\mathrm{b}}=0.3$ to $\Gamma_{\mathrm{b}}=3.3$, which can lead to some saturation for high IMF values.

Given the CALIFA wavelength range $(\lambda \lambda 3700,7500 \AA)$, we focused on five prominent spectral indices: $\mathrm{H}_{\beta_{O}},[\mathrm{MgFe}]^{\prime}$, $\mathrm{Mg} 2 \mathrm{Fe}$ (Bruzual \& Charlot 2003), $\mathrm{NaD}, \mathrm{TiO}_{2_{\text {CALIFA }}}$, and $\mathrm{TiO}_{1}$. The last three are IMF-sensitive features, whereas $[\mathrm{MgFe}]^{\prime}$ and $\mathrm{Mg} 2 \mathrm{Fe}$ depend on metallicity and $\mathrm{H}_{\beta_{o}}$ on age. The $\mathrm{TiO}_{2_{\text {CALIFA }}}$ index follows the standard $\mathrm{TiO}_{2}$ definition (Trager et al. 1998) but with a narrower blue pseudo-continuum ( $\lambda \lambda$ 6060.625, $6080.625 \AA$ ) to avoid any telluric contamination. The correction of $\mathrm{H}_{\beta_{o}}$ from nebular emission was done in the same way as in La Barbera et al. (2013). For each spectrum (i.e., each galaxy and radial bin), we inferred stellar population properties with three different approaches.

(i) Following La Barbera et al. (2013), we minimized

$$
\begin{aligned}
& \chi^{2}\left(\Gamma_{\mathrm{b}}, \text { age, }[\mathrm{M} / \mathrm{H}]\right) \\
& \quad=\sum_{i}\left[\frac{\left(\mathrm{EW}_{i}-\Delta_{\alpha, i}\right)-\mathrm{EW}_{M, i}}{\sigma_{\mathrm{EW}_{i}}}\right]^{2},
\end{aligned}
$$

where our index measurements $\left(\mathrm{EW}_{i}\right)$, after being corrected for non-solar abundances $\left(\Delta_{\alpha, i}\right)$, are compared to the model predictions $\left(\mathrm{EW}_{M, i}\right)$. The fitting process was repeated by considering different combinations of IMF sensitive indices, leading to consistent results.

(ii) We implemented an iterative fitting scheme where age and metallicity were calculated first using an index-index $\left(\mathrm{H}_{\beta_{o}}-[\mathrm{MgFe}]^{\prime}\right)$ diagram, assuming a standard IMF. The age estimate coming from this first step was then used to derive the metallicity and the IMF in a second indexindex $\left(\mathrm{TiO}_{2_{\text {CALIFA }}}[\mathrm{MgFe}]^{\prime}\right)$ diagram. These two steps were repeated, re-deriving age and metallicity from the $\mathrm{H}_{\beta_{o}}-$ $[\mathrm{MgFe}]^{\prime}$ diagram with an updated IMF, until the solution converged.

(iii) Alternatively, we also fit our data following Equation (1) but neglecting individual $[\alpha / \mathrm{Fe}]$ corrections.

The three approaches show a good agreement, with small differences only for low IMF-slope values, where IMF effects can be mimicked by changes in other stellar population parameters. For simplicity, we will refer through this Letter to the best-fitting values derived from the simultaneous fit (Equation (1)) of the $\mathrm{H}_{\beta_{o}},[\mathrm{MgFe}]^{\prime}$ and $\mathrm{TiO}_{2 \text { CALIFA }}$ indices. Note that the latter, compared to the $\mathrm{TiO}_{1}$, exhibits a milder dependence on both abundance ratios and total metallicity (La Barbera et al. 2013). However, the absolute $\mathrm{TiO}_{2}$ sensitivity to these parameters depends on the adopted stellar population synthesis model (Bruzual \& Charlot 2003; Thomas et al. 2011). Moreover, $\mathrm{TiO}_{2}$ is less sensitive to variations in the temperature scale of giant stars. Spiniello et al. (2014b) showed that, after accounting for metallicity, no variation in the effective temperature is needed to fit the strength of gravitysensitive features in massive ETGs, and that a similar result would be recovered with other stellar population models if the same IMF parameterization were used. Although our fitting scheme does not account for the residual impact of non-solar abundances on the temperature scale of the (solar-scaled) isochrones, the $[\alpha / \mathrm{Fe}]$ effect on the isochrones is significantly milder than that on the stellar atmospheres (Vazdekis et al. 2015). The latter is corrected in our approach $\left(\Delta_{\alpha, i}\right.$ in Equation (1)).

In addition to the IMF, age and metalliciy, the $[\mathrm{Mg} / \mathrm{Fe}]$ of each radial bin was derived by means of the $\left[Z_{\mathrm{Mg}} / Z_{\mathrm{Fe}}\right]$ proxy (La Barbera et al. 2013), i.e., using the metallicity difference between two index-index diagrams, where $\mathrm{H}_{\beta_{O}}$ is plotted against a $\mathrm{Mg}$ and $\mathrm{Fe}$ metallicity indicator, respectively.

\subsection{Stellar Kinematics}

To understand the IMF variations, we also compared our best-fitting IMF values to two kinematics parameters: the local $\sigma$ and the local $V_{\text {rms }}$ defined as $V_{\text {rms }} \equiv \sqrt{V^{2}+\sigma^{2}}$. Whereas $\sigma$ has been claimed to be the main driver of the IMF variations (Treu et al. 2010; Ferreras et al. 2013), in spatially resolved studies $V_{\text {rms }}$ accounts for both random and ordered motions.

\section{RESULTS}

In Figure 1 we present the correlation between the bestfitting IMF slope, $\Gamma_{\mathrm{b}}$, and the local values of $[\mathrm{Mg} / \mathrm{Fe}]$, age, $\sigma$, and $V_{\text {rms }}$. None of them show a tight correlation with $\Gamma_{\mathrm{b}}$. The mild relation between age and $\Gamma_{\mathrm{b}}$ can be understood either as a residual degeneracy between both parameters or as a consequence of the IMF-metallicity relation, since young stars within massive ETGs are likely formed from metal-enriched material.

\subsection{The IMF-Metallicity Relation}

Among all the explored relations, the IMF slope-local metallicity relation emerges as the most fundamental. This is shown in Figure 2, where the local $\Gamma_{b}-[\mathrm{M} / \mathrm{H}]$ relation derived from the CALIFA survey (blue) is combined with local IMF estimates, obtained at different galactocentric distances, for three nearby ETGs by Martín-Navarro et al. (2015a, 2015b; red and orange symbols). In addition, we also show (black) the best-fitting IMF and metallicity inferred from SDSS stacked spectra. To construct these spectra, we followed La Barbera et al. (2013), but binning according to both $\sigma$ and $[\mathrm{M} / \mathrm{H}]$ of the individual galaxies. The broad wavelength range of the SDSS data set allows us to infer the IMF, not only using those features within the CALIFA spectral range, but also prominent near-IR IMF sensitive features such as the $\mathrm{NaI} 8189$ and the CaII triplet (see Section 4.1 in La Barbera et al. 2013 for a 

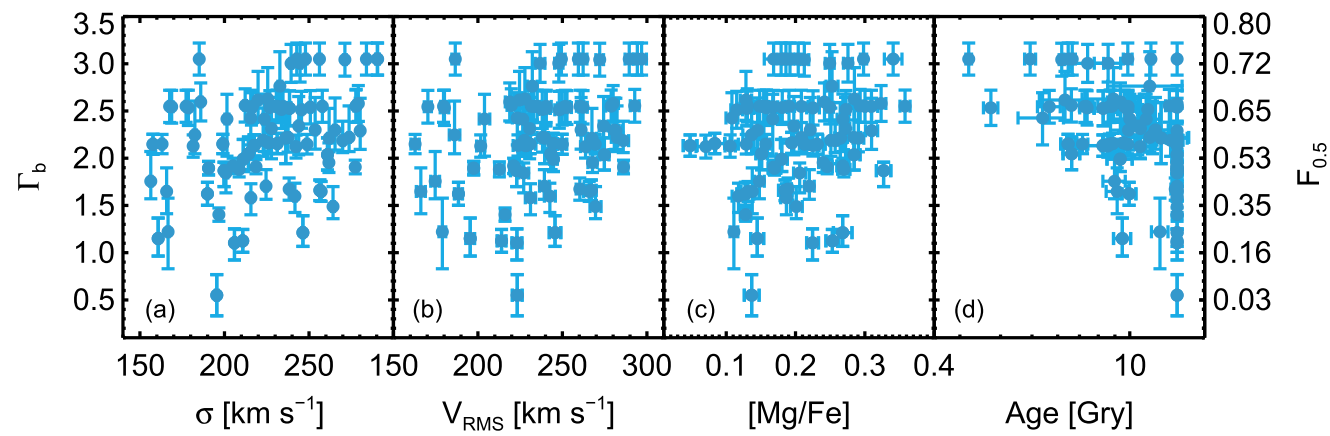

Figure 1. Best-fitting IMF slope $\Gamma_{\mathrm{b}}$ is compared to the local $\sigma(\mathrm{a}), V_{\mathrm{rms}}(\mathrm{b}),[\mathrm{Mg} / \mathrm{Fe}](\mathrm{c})$, and age (b). Neither the kinematics properties nor the $[\mathrm{Mg} / \mathrm{Fe}]$ or the age follow the measured IMF variations $\left(\rho_{\sigma}=0.35, \rho_{V_{\mathrm{rms}}}=0.30, \rho_{[\mathrm{Mg} / \mathrm{Fe}]}=0.21, \rho_{\mathrm{age}}=-0.50\right.$, with $\rho$ being the Spearman correlation coefficient). The right vertical axis represents the IMF slope in terms of $F_{0.5}$, defined as the fraction (with respect to the total mass) of stars with masses below $0.5 M_{\odot}$.

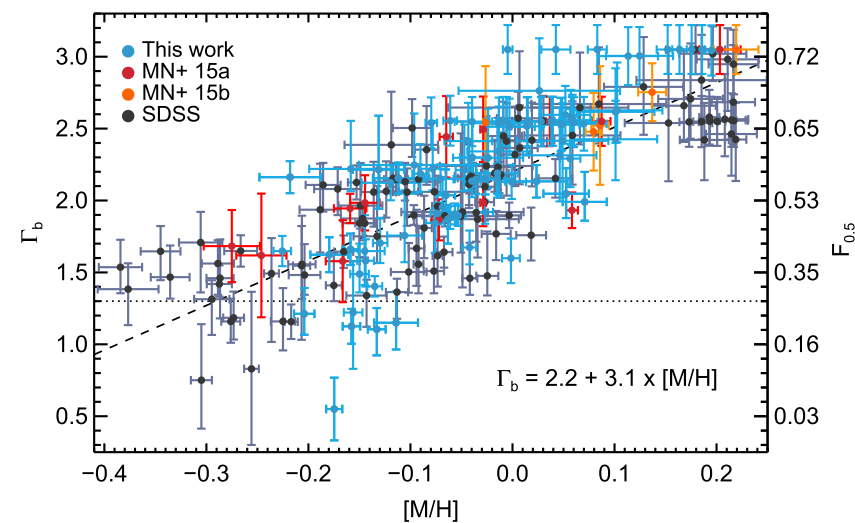

Figure 2. IMF-metallicity relation obtained from CALIFA local measurements (blue). We also show the local IMF and metallicities measurements derived by Martín-Navarro et al. (2015a, 2015b; red, orange) for three of nearby ETGs, as well as global SDSS measurements (black). We found it to be the strongest correlation $\left(\rho_{[\mathrm{M} / \mathrm{H}]}=0.82\right)$. As in Figure 1, the right vertical axis indicates the $F_{0.5}$ ratio. For reference, the standard Kroupa IMF value is shown as a horizontal dotted line. Dashed line correspond to the best-fitting linear relation to all the data sets.

detailed description of gravity-sensitive features in SDSS spectra).

The fact that the three data sets included, although based on different sets of line-strengths, lie on the same relation, supports a tight connection between IMF slope and metallicity, regardless of the details in the determination of the stellar population parameters. Moreover, the agreement between integrated measurements from the SDSS spectra and spatially resolved values, suggests that the mechanism behind the local IMF variations ultimately shapes the global galaxy mass-IMF relation.

A linear fit to all the measurements shown in Figure 2 leads to the following relation between IMF slope and metallicity in ETGs:

$$
\Gamma_{\mathrm{b}}=2.2( \pm 0.1)+3.1( \pm 0.5) \times[\mathrm{M} / \mathrm{H}]
$$

Since IMF-sensitive features ultimately trace the dwarf-to-giant ratio $F_{0.5}$, as defined in La Barbera et al. (2013), the above equation can be expressed in terms of a single power law IMF as

$$
\Gamma=1.50( \pm 0.05)+2.1( \pm 0.2) \times[\mathrm{M} / \mathrm{H}] .
$$

Apart from the measurement errors, the scatter in the relation comes from two sources: the IMF- $[\alpha / \mathrm{Fe}]$ degeneracy when fitting gravity-sensitive features around the Kroupa-like IMF regime (La Barbera et al. 2013) and the dependence of the IMF

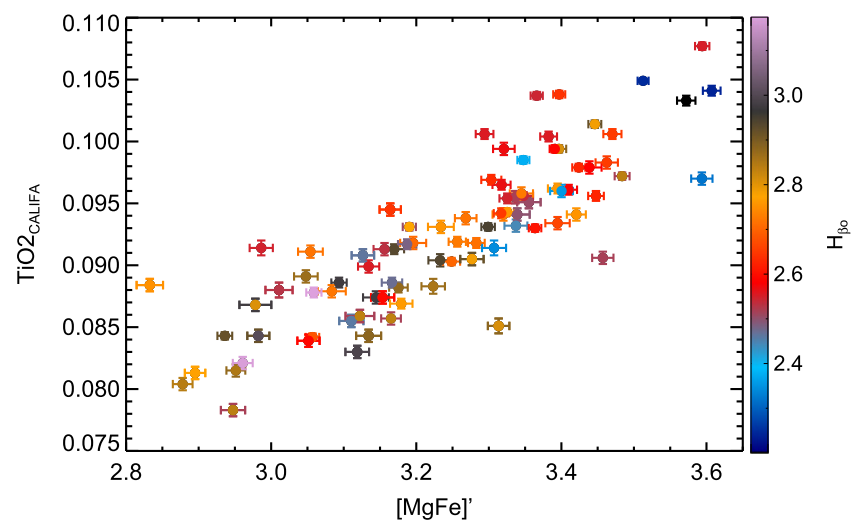

Figure 3. Empirical relation between the metallicity-sensitive $[\mathrm{MgFe}]^{\prime}$ and the IMF-sensitive $\mathrm{TiO}_{2 \text { CALIFA }}$ features. Index measurements (at a resolution of $200 \mathrm{~km} \mathrm{~s}^{-1}$ ) are color-coded by their $\mathrm{H}_{\beta_{o}}$ value, as an age proxy. An IMFmetallicity relation is needed to explain the observed trend, since the $\mathrm{TiO}_{2 \text { CALIFA }}$ weakly depends on the total metallicity and $[\mathrm{MgFe}]^{\prime}$ is almost independent of the IMF.

on the minimized set of indices ${ }^{12}$ (Spiniello et al. 2014a). In this sense, the fact that the $\mathrm{TiO}_{2}$-based CALIFA measurements show a steeper IMF-metallicity trend is consistent with a stronger metallicity dependence of this index than predicted in the MILES models. On the other hand, the consistency among different data sets $(\rho=0.82$ when CALIFA, SDSS and Martín-Navarro et al. 2015a, 2015b are considered) points to a genuine IMF- $[\mathrm{M} / \mathrm{H}]$ trend, as shown in Figure 2.

\subsection{The $[\mathrm{MgFe}]^{\prime}-\mathrm{TiO}_{2_{\mathrm{CALIFA}}}$ Empirical Relation}

To strengthen the validity of our result, we adopted an empirical approach. In Figure 3 we compare the $[\mathrm{MgFe}]^{\prime}$ to the $\mathrm{TiO}_{2_{\text {CALIFA }}}$ individual measurements for all radial bins in our sample, at a common $200 \mathrm{~km} \mathrm{~s}^{-1}$ resolution. Each point is color-coded by its $\mathrm{H}_{\beta_{O}}$ value, representing an age segregation. The $[\mathrm{MgFe}]^{\prime}$ index is independent of the IMF, depending almost entirely on the total metallicity. On the contrary, the $\mathrm{TiO}_{2_{\text {CALIFA }}}$ is a strong IMF indicator, that increases with age $(\mathrm{La}$ Barbera et al. 2013). Since MILES stellar population models predict the $\mathrm{TiO}_{2_{\text {CALIFA }}}$ feature to be, in the explored metallicity and age regime, almost independent of total metallicity, the strong (correlation coefficient $\rho=0.86$ ) correlation shown in Figure 3 can be interpreted as a metallicity-IMF trend. A

\footnotetext{
12 Uncertainties in Equations (2) and (3) account for this effect, by repeating the fit using only CALIFA data.
} 
similar relation was found by Trager et al. (1998) while comparing the $\mathrm{Mg}_{2}$ and the $\mathrm{TiO}_{2}$ indices. Note that if the $\mathrm{TiO}_{2}$ sensitivity to total metallicity is larger than predicted by MILES models, a certain correlation between these two indices would also be expected.

\section{DISCUSSION}

ETGs are characterized by old stellar populations and thus, only stars with masses $M \lesssim 1 M_{\odot}$ remain alive. Therefore, the analysis of gravity-sensitive features in the integrated light of unresolved ETGs can only constraint the the dwarf-to-giant ratio. We vary this ratio by changing the slope $\left(\Gamma_{b}\right)$ of the highmass end of the IMF, while it was kept constant for stars with masses below $0.5 M_{\odot}$. Notice that our results, restricted to inference of the dwarf-to-giant ratio, barely depend on the detailed IMF shape, but it must be considered when exploring, for example, the chemical evolution and the expected mass-tolight ratios of ETGs.

\subsection{The Underlying Parameters Behind the Varying Dwarf-to-Giant Ratio in ETGs}

Two competing candidates have been proposed to explain the observed dwarf-to-giant ratio variations in ETGs: $\sigma$ and $[\mathrm{Mg} / \mathrm{Fe}]$. Smith (2014) investigated these two parameters over the same sample of galaxies (Cappellari et al. 2011; Conroy \& van Dokkum 2012), pointing out that the stellar population analysis favors the stellar population property $([\mathrm{Mg} / \mathrm{Fe}])$, whereas a dynamical analysis supports the dynamical-related quantity $(\sigma)$. The work of Smith opened the question of whether IMF studies are actually probing the IMF or whether they are, at least partially, dominated by confounding factors. However, stellar population analyses of SDSS stacked spectra (La Barbera et al. 2013; Spiniello et al. 2014a) have shown a strong correlation between $\sigma$ and the dwarf-to-giant ratio. Moreover, La Barbera et al. (2015), using also SDSS stacked spectra, showed that $[\mathrm{Mg} / \mathrm{Fe}]$ is loosely (or un-) correlated with the IMF slope. The latter result is in agreement with panel (c) in Figure 1, where we show that the local $[\mathrm{Mg} / \mathrm{Fe}]$ is decoupled from the dwarf-to-giant ratio variations.

Regarding $\sigma$, in Martín-Navarro et al. (2015b) we showed that its local value was not the main driver behind the dwarf-togiant ratio variations of the massive relic galaxy NGC 1277. In the present work we confirm this result using a statistically significant sample of ETGs (panel (a) in Figure 1). Notice that previous studies suggesting a connection between $\sigma$ and the IMF slope were based on spatially unresolved spectra. Thus, their velocity dispersions trace the overall galaxy potential (mass) rather than the detailed kinematics. Thanks to the CALIFA data, we propose that this connection between galaxy mass and IMF slope arises from considering simultaneously both the galaxy mass-metallicity and the metallicity-IMF slope relations. In this sense, the dwarf-to-giant ratio gradients observed in ETGs (Martín-Navarro et al. 2015a, 2015b) can be also accounted for the radial metallicity variation within these galaxies. To illustrate the suggested metallicity-driven galaxy mass-IMF slope relation, we used the above described Sloan data (black dots in Figure 2) to calculate the mean global metallicity per galaxy mass $(\sigma)$ bin. Then, we transformed this metallicity into the expected IMF slope according to Equation (2). The result is summarized in Figure 4. Although it can not be directly compared with previous works, we qualitatively

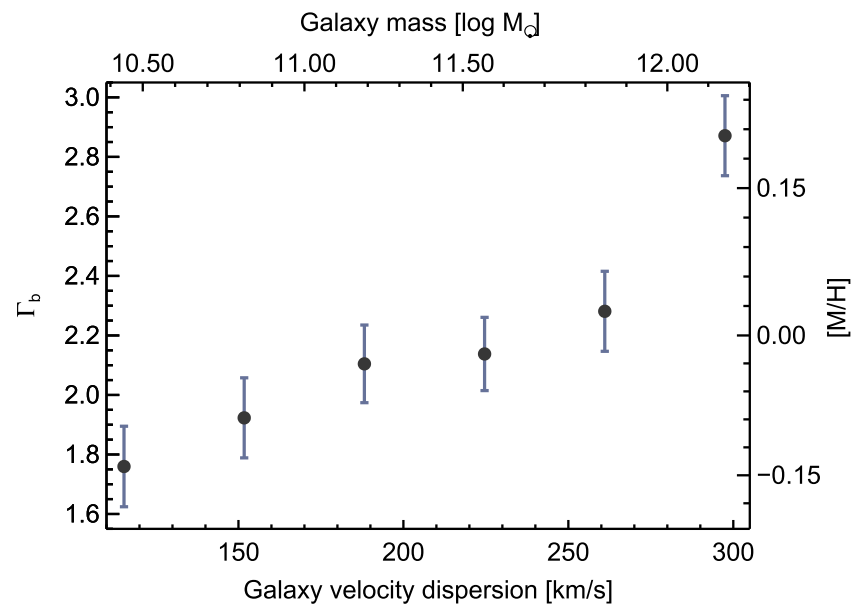

Figure 4. Predicted IMF slope-galaxy velocity dispersion relation. The bestfitting metallicity of the global SDSS measurements (right vertical axis) was transformed, following Equation (2), into an expected IMF-slope value $\Gamma_{\mathrm{b}}$. The upper horizontal axis represents the galaxy mass, estimated from the velocity dispersion (Cappellari et al. 2013). We suggest that the relation found between IMF slope and galaxy mass can be understood as the combination of the massmetallicity and the metallicity-IMF slope relations.

recover the observed relation between galaxy mass and IMF slope, with more massive galaxies being bottom-heavier.

\subsection{Metallicity as a Driver of IMF Variations}

Metallicity-driven dwarf-to-giant ratio variations can be explained if molecular clouds collapse following a Jeans spectrum. Such a scenario is expected if thermal pressure dominates the process, although a turbulent medium can yield similar results if dust cooling is significant (Larson 2005). Perhaps, a realistic scenario would couple a Jeans-driven mechanism with turbulence-induced fragmentation (e.g., Padoan \& Nordlund 2002). Nevertheless, this Letter shows the importance that metallicity plays in determining the stellar mass spectrum. Such a scenario is consistent with studies of nearby resolved galaxies (Geha et al. 2013), line-strength indices (Cenarro et al. 2003) and optical colors (Ricciardelli et al. 2012). In low-metallicity regions, it is found that molecular clouds are very efficient in forming massive stars, leading to top-heavy IMFs. This is supported by observations of metal-poor systems showing evidence of an IMF dominated by massive stars (Marks et al. 2012).

If metallicity is one of the main drivers behind the IMF variations, a number of fundamental aspects of galaxy evolution must be revisited. Because the stellar feedback regulates the chemical enrichment of galaxies, IMF, star formation and chemical composition will be tightly related during the time evolution. In particular, massive galaxies are expected to increase their metallicity during the formation of their stellar populations, and therefore, the dwarf-to-giant ratio should have been smaller at higher redshifts, such that there were fewer low-mass stars per high-mass star. Such a timedependent IMF scenario (Arnaud et al. 1992; Vazdekis et al. 1997; Larson 1998; Weidner et al. 2013; Ferreras et al. 2015) would explain the observations of nearby massive ETGs, which are inconsistent with a time-invariant, steep (or even Milky Way like) IMF slope (Arrigoni et al. 2010).

Moreover, the $[\mathrm{Mg} / \mathrm{Fe}]$ has been extensively used as a proxy for the formation time-scale (e.g., Thomas et al. 2005): rapid formation events lead to high $[\mathrm{Mg} / \mathrm{Fe}]$ values. However, an 
IMF dominated at early times by high-mass stars would also produce an enhanced $[\mathrm{Mg} / \mathrm{Fe}]$, relaxing the typical $(\sim 1 \mathrm{Gyr})$ constraint on the rapid formation time-scale. Thus, to safely interpret $z \sim 0$ observations, it is crucial to understand the chemical evolution of galaxies since they were formed, and in particular, during the build-up of their chemical properties (Vazdekis et al. 1996). In this sense, it has been shown (e.g., Ferreras et al. 2009; Choi et al. 2014; Ferré-Mateu et al. 2014) that the chemical composition of massive galaxies has remained constant over the last $\sim 7$ Gyr. If metallicity actually regulates the dwarf-to-giant ratio variations, it would imply that the IMF of massive objects was bottom-heavy at $z \sim 1$, as recently suggested by observations (Shetty \& Cappellari 2014; Martín-Navarro et al. 2015c), since no chemical evolution has happened since then.

Finally, we want to emphasize that the present study is restricted to the analysis of ETGs. The local conditions (e.g., turbulence, pressure, density, radiation, and magnetic fields) of the interstellar medium at high- $z$, or even in nearby disk galaxies, are expected to differ from the local environment within ETGs. Thus, more observational efforts are still needed to fully characterize the IMF behavior with varying star-forming conditions. In fact, for very metal-poor systems, Geha et al. (2013) found a slightly top-heavy IMF, but steeper than expected from a crude extrapolation of Equation (2). The trend in Figure 2 seems to actually flatten at lowest and highest metallicity, pointing to a nonlinearity of the IMF-metallicity relation.

\section{SUMMARY}

Although deviations from the standard Milky Way IMF have been extensively reported in the literature over the last years, the mechanism responsible for these variations remains unknown. We have analyzed the radial gradients of IMFsensitive features in a sample of 24 ETGs observed by the CALIFA survey, finding that the local IMF is tightly related to the local metallicity. Our result agrees with previous works reporting local and global IMF variations, and it explains the observed galaxy mass-IMF relation. We speculate about the implications of metallicity-driven IMF variations in the context of galaxy formation and evolution.

The intimate connection between IMF and metallicity described in our work suggests a complex massive galaxy formation process, departing from the classical picture where these objects formed under nearly stationary conditions. Thus, to safely understand and interpret $z \sim 0$ observations, it is necessary to untangle the early life of massive galaxies at high$z$. The combination of more sophisticated stellar population synthesis models (Conroy \& van Dokkum 2012; Vazdekis et al. 2015) and high- $z$ spectroscopic surveys (Brammer et al. 2012; Kriek et al. 2014) will provide valuable insights.

We acknowledge support from grants AYA2013-48226-C3$1 \mathrm{P}$ and AYA2010-15081 from the Spanish MINECO. C.J.W. acknowledges support through the Marie Curie Career Integration Grant 303912. P.S.-B. acknowledges support from the Ramón y Cajal program, ATA2010-21322-C03-02 (MINECO). J.F.B. and Gvd.V. acknowledge the DAGAL network from the People Programme (Marie Curie Actions) of the European Unions Seventh Framework Programme FP7/ 2007-2013 under REA grant agreement number PITN-GA2011-289313.

\section{REFERENCES}

Arnaud, M., Rothenflug, R., Boulade, O., Vigroux, L., \& Vangioni-Flam, E. 1992, A\&A, 254, 49

Arrigoni, M., Trager, S. C., Somerville, R. S., \& Gibson, B. K. 2010, MNRAS, 402, 173

Bastian, N., Covey, K. R., \& Meyer, M. R. 2010, ARA\&A, 48, 339

Brammer, G. B., van Dokkum, P. G., Franx, M., et al. 2012, ApJS, 200, 13

Bruzual, G., \& Charlot, S. 2003, MNRAS, 344, 1000

Cappellari, M., Emsellem, E., Krajnović, D., et al. 2011, MNRAS, 413, 813

Cappellari, M., McDermid, R. M., Alatalo, K., et al. 2012, Natur, 484, 485

Cappellari, M., McDermid, R. M., Alatalo, K., et al. 2013, MNRAS, 432, 1862

Cenarro, A. J., Gorgas, J., Vazdekis, A., Cardiel, N., \& Peletier, R. F. 2003, MNRAS, 339, L12

Choi, J., Conroy, C., Moustakas, J., et al. 2014, ApJ, 792, 95

Conroy, C., \& van Dokkum, P. G. 2012, ApJ, 760, 71

Ferré-Mateu, A., Sánchez-Blázquez, P., Vazdekis, A., \& de la Rosa, I. G. 2014, ApJ, 797, 136

Ferreras, I., La Barbera, F., de la Rosa, I. G., et al. 2013, MNRAS, 429, L15

Ferreras, I., Pasquali, A., Malhotra, S., et al. 2009, ApJ, 706, 158

Ferreras, I., Weidner, C., Vazdekis, A., \& La Barbera, F. 2015, MNRAS, in press (arXiv:1501.01636)

García-Benito, R., Zibetti, S., Sánchez, S. F., et al. 2014, A\&A, submitted (arXiv:1409.8302)

Geha, M., Brown, T. M., Tumlinson, J., et al. 2013, ApJ, 771, 29

González Delgado, R. M., Pérez, E., Cid Fernandes, R., et al. 2014, A\&A, 562, A47

Graham, A. W., Trujillo, I., \& Caon, N. 2001, AJ, 122, 1707

Kriek, M., Shapley, A. E., Reddy, N. A., et al. 2014, ApJS, in press (arXiv:1412.1835)

Kroupa, P. 2002, Sci, 295, 82

La Barbera, F., Ferreras, I., \& Vazdekis, A. 2015, MNRAS, 449, L137

La Barbera, F., Ferreras, I., Vazdekis, A., et al. 2013, MNRAS, 433, 3017

Larson, R. B. 1998, MNRAS, 301, 569

Larson, R. B. 2005, MNRAS, 359, 211

Marks, M., Kroupa, P., Dabringhausen, J., \& Pawlowski, M. S. 2012, MNRAS, 422, 2246

Martín-Navarro, I., Barbera, F. L., Vazdekis, A., Falcón-Barroso, J., \& Ferreras, I. 2015, MNRAS, 447, 1033

Martín-Navarro, I., La Barbera, F., Vazdekis, A., Falcón-Barroso, J., \& Ferreras, I. 2015, MNRAS, 451, 1081

Martín-Navarro, I., Pérez-González, P. G., Trujillo, I., et al. 2015, ApJL, 798, L4

Padoan, P., \& Nordlund, Å 2002, ApJ, 576, 870

Pastorello, N., Forbes, D. A., Foster, C., et al. 2014, MNRAS, in press (arXiv:1405.2338)

Ricciardelli, E., Vazdekis, A., Cenarro, A. J., \& Falcón-Barroso, J. 2012, MNRAS, 424, 172

Sánchez, S. F., Kennicutt, R. C., Gil de Paz, A., et al. 2012, A\&A, 538, A8

Shetty, S., \& Cappellari, M. 2014, ApJL, 786, L10

Smith, R. J. 2014, MNRAS, in press (arXiv:1403.6114)

Spiniello, C., Trager, S., Koopmans, L. V. E., \& Conroy, C. 2014, MNRAS, 438, 1483

Spiniello, C., Trager, S. C., \& Koopmans, L. V. E. 2014, ApJ, in press (arXiv:1401.1145)

Spiniello, C., Trager, S. C., Koopmans, L. V. E., \& Chen, Y. P. 2012, ApJL, 753, L32

Thomas, D., Maraston, C., Bender, R., \& Mendes de Oliveira, C. 2005, ApJ, 621,673

Thomas, J., Saglia, R. P., Bender, R., et al. 2011, MNRAS, 415, 545

Trager, S. C., Worthey, G., Faber, S. M., Burstein, D., \& Gonzalez, J. J. 1998, ApJS, 116, 1

Treu, T., Auger, M. W., Koopmans, L. V. E., et al. 2010, ApJ, 709, 1195

van Dokkum, P. G., \& Conroy, C. 2010, Natur, 468, 940

Vazdekis, A., Casuso, E., Peletier, R. F., \& Beckman, J. E. 1996, ApJS, 106,307

Vazdekis, A., Coelho, P., Cassisi, S., et al. 2015, MNRAS, 449, 1177

Vazdekis, A., Peletier, R. F., Beckman, J. E., \& Casuso, E. 1997, ApJS, 111,203

Vazdekis, A., Ricciardelli, E., Cenarro, A. J., et al. 2012, MNRAS, 424, 157

Vazdekis, A., Sánchez-Blázquez, P., Falcón-Barroso, J., et al. 2010, MNRAS, 404, 1639

Walcher, C. J., Wisotzki, L., Bekeraité, S., et al. 2014, A\&A, 569, A1

Weidner, C., Ferreras, I., Vazdekis, A., \& La Barbera, F. 2013, MNRAS, 435,2274 